\title{
Galaxy cluster angular-size data constraints on dark energy
}

\author{
Y. Chen ${ }^{1,2}$ and B. Ratra ${ }^{1}$ \\ ${ }^{1}$ Department of Astronomy, Beijing Normal University, 100875 Beijing, PR China \\ e-mail: chenyun@mail . bnu.edu.cn \\ 2 Department of Physics, Kansas State University, 116 Cardwell Hall, Manhattan, KS 66506, USA \\ e-mail: ratra@phys.ksu.edu
}

Received 25 August 2011 / Accepted 20 April 2012

\begin{abstract}
We use angular size versus redshift data for galaxy clusters provided by Bonamente and collaborators to place constraints on model parameters of constant and time-evolving dark energy cosmological models. These constraints are compatible with those from other recent data, but are not very restrictive. A joint analysis of the galaxy cluster angular-size data with the more restrictive baryon acoustic oscillation peak length scale and supernova Type Ia apparent-magnitude data, favors a spatially flat cosmological model currently dominated by a time-independent cosmological constant, but does not exclude time-varying dark energy.
\end{abstract}

Key words. cosmology: miscellaneous - cosmology: theory - dark energy

\section{Introduction}

A number of lines of observational evidence support a "standard" model of cosmology with an energy budget that is significantly dominated by dark energy. Dark energy is most simply characterized as a negative-pressure substance that powers the observed accelerated cosmological expansion. It can evolve slowly in time and vary weakly in space, although current data are consistent with it being equivalent to Einstein's cosmological constant. On the other hand, some studies argue that the observed accelerated expansion should instead be viewed as an indication that general relativity does not accurately describe gravitational physics on large cosmological length scales. For relevant reviews, we refer to Frieman (2008), Ratra \& Vogeley (2008), Caldwell \& Kamionkowski (2009), Sami (2009), Bartelmann (2010), Cai et al. (2010), and Brax (2009). In what follows, we assume that general relativity provides an accurate description of gravitation on cosmological length scales.

There are many dark energy models under discussion. For recent discussions, we cite the studies of Wei (2011), Jamil \& Saridakis (2010), Maggiore (2011), Dutta \& Scherrer (2010), Shao \& Chen (2010), Lepe \& Peña (2010), Sloth (2010), Liu (2010), Honorez et al. (2010), and references therein. Perhaps the most economical - and the current "standard" model - is the $\Lambda$ CDM model (Peebles 1984), where the accelerated cosmological expansion is powered by Einstein's cosmological constant, $\Lambda$, a spatially homogeneous fluid with equation of state parameter $\omega_{\Lambda}=p_{\Lambda} / \rho_{\Lambda}=-1$ (with $p_{\Lambda}$ and $\rho_{\Lambda}$ being the fluid pressure and energy density). In this model, the cosmological energy budget is dominated by $\rho_{\Lambda}$, with cold dark matter (CDM) being the second largest contributor. The $\Lambda \mathrm{CDM}$ model provides a reasonable fit to most observational constraints, although the "standard" CDM structure formation model might be in some observational trouble (see, e.g., Peebles \& Ratra 2003; Perivolaropoulos 2010).
The $\Lambda$ CDM model has a few apparent puzzles. Prominent among these is that the needed $\Lambda$ energy density scale is of order an meV, very small compared to the higher (cutoff) value suggested by a perhaps naive application of quantum mechanics. Another puzzle is that we happen to be observing at a time not very different from when the $\Lambda$ energy density started dominating the cosmological energy budget (this is the "coincidence" puzzle).

If the dark energy density - that responsible for powering the accelerated cosmological expansion - slowly decreased in time (rather than remaining constant like $\rho_{\Lambda}$ ), the energy densities of dark energy and nonrelativistic matter (CDM and baryons) would remain comparable for a longer period of time, and so alleviate the coincidence puzzle. In addition, a slowly decreasing dark energy density that is based on more fundamental physics at an energy density scale much higher than an $\mathrm{meV}$, would result in a current dark energy density scale of an meV through gradual decrease over the long lifetime of the Universe. Thus, a slowly decreasing dark energy density could resolve some of the puzzles of the $\Lambda$ CDM model (Ratra \& Peebles 1988).

The XCDM parametrization is often used to describe a slowly decreasing dark energy density. In this parametrization, the dark energy is modeled as a spatially homogeneous $(X)$ fluid with an equation of state parameter $w_{X}=p_{X} / \rho_{X}$, where $w_{X}<-1 / 3$ is an arbitrary constant and $p_{X}$ and $\rho_{X}$ are the pressure and energy density of the $X$-fluid. When $w_{X}=-1$, the $\mathrm{XCDM}$ parametrization reduces to the $\Lambda \mathrm{CDM}$ model, which is a complete and consistent model. For any other value of $w_{X}(<-1 / 3)$, the XCDM parametrization is incomplete as it cannot describe spatial inhomogeneities (see, e.g., Ratra 1991). For computational simplicity, we study the XCDM parametrization only in the spatially flat cosmological case.

If the dark energy density evolves in time, physics demands that it also be spatially inhomogeneous. The $\phi \mathrm{CDM}$ model in which dark energy is modeled as a scalar field $\phi$ with a 
gradually decreasing (in $\phi$ ) potential-energy density $V(\phi)-$ is the simplest complete and consistent model of a slowly decreasing (in time) dark energy density. Here we focus on an inverse power-law potential-energy density $V(\phi) \propto \phi^{-\alpha}$, where $\alpha$ is a nonnegative constant (Peebles \& Ratra 1988; Ratra \& Peebles 1988). When $\alpha=0$, the $\phi \mathrm{CDM}$ model reduces to the corresponding $\Lambda \mathrm{CDM}$ case. Here we only consider the spatially flat $\phi \mathrm{CDM}$ cosmological model.

It has been known for some time that a spatially flat $\Lambda C D M$ model with a current energy budget dominated by a constant $\Lambda$ is largely consistent with most observational constraints (see, e.g., Jassal et al. 2010; Wilson et al. 2006; Davis et al. 2007; Allen et al. 2008). Supernovae Type Ia (SNeIa) apparentmagnitude measurements (e.g., Riess et al. 1998; Perlmutter et al. 1999; Shafieloo et al. 2009; Holsclaw et al. 2010), in conjunction with cosmic microwave background (CMB) anisotropy data (e.g., Ratra et al. 1999; Podariu et al. 2001b; Spergel et al. 2003; Komatsu et al. 2009, 2011) combined with low estimates of the cosmological mass density (e.g., Chen \& Ratra 2003b), as well as baryon acoustic oscillation (BAO) peak length scale estimates (e.g., Percival et al. 2007; Gaztañaga et al. 2009; Samushia \& Ratra 2009b; Wang 2009) strongly suggest that we live in a spatially flat $\Lambda \mathrm{CDM}$ model with nonrelativistic matter contributing a little less than $30 \%$ of the current cosmological energy budget, with the remaining slightly more than $70 \%$ being contributed by a cosmological constant. These three sets of data carry by far the most weight when determining constraints on models and cosmological parameters.

Future data from space missions will tighten the constraints (see, e.g., Podariu et al. 2001a; Samushia et al. 2011; Wang et al. 2010). However, at present, it is of great importance to consider independent constraints that can be derived from other presently available data sets. While these data do not yet carry as much statistical weight as the SNeIa, CMB, and BAO data, they can potentially reassure us (if they provide constraints consistent with those from the better known data), or if the two sets of constraints are inconsistent this might lead to the discovery of hidden systematic errors or rule out the cosmological model under consideration.

Other data that have been used to constrain cosmological parameters include galaxy cluster gas-mass fraction (e.g., Allen et al. 2008; Samushia \& Ratra 2008; Ettori et al. 2009), gammaray burst luminosity distance (e.g., Schaefer 2007; Liang \& Zhang 2008; Wang 2008; Samushia \& Ratra 2010), large-scale structure (e.g., Courtin et al. 2011; Baldi 2011; Basilakos et al. 2010), strong gravitational lensing (e.g., Chae et al. 2002, 2004; Lee \& Ng 2007; Yashar et al. 2009), and lookback time (e.g., Capozziello et al. 2004; Simon et al. 2005; Samushia et al. 2010; Dantas et al. 2011) or Hubble parameter (Samushia \& Ratra 2006; Samushia et al. 2007; Fernandez-Martinez \& Verde 2008; Yang \& Zhang 2010) data. While the constraints provided by these data are much less restrictive than those derived from the $\mathrm{SNeIa}, \mathrm{CMB}$, and BAO data, both types of data result in largely compatible constraints that generally support a currently accelerating cosmological expansion. This gives us confidence that the broad outlines of the "standard" cosmological model are now in place.

Angular-size data from compact radio sources have also been used to constrain cosmological parameters (see, e.g., Gurvits et al. 1999; Guerra et al. 2000; Lima \& Alcaniz 2000; Lima \& Alcaniz 2002; Chen \& Ratra 2003a; Podariu et al. 2003; Santos \& Lima 2008). There are two very recent samples of angular size versus redshift data from galaxy clusters obtained by combining their Sunyaev-Zeldovich effect (SZE) and X-ray surface brightness observations. These are the 25 data points from De Filippis et al. (2005) and the 38 data points from Bonamente et al. (2006). De Filippis et al. (2005) obtained their angular-size data by using an isothermal elliptical model for the galaxy clusters, while Bonamente et al. (2006) derived their data by assuming a non-isothermal spherical model. The sample of De Filippis et al. (2005) was used to constrain $H_{0}$, result in good agreement with the independent studies of the Hubble Space Telescope key project and the estimates of WMAP (Cunha et al. 2007). The sample of Bonamente et al. (2006) has been previously used to constrain some cosmological parameters and to test the distance duality relationship of metric gravity models (see, e.g., De Bernardis et al. 2006; Holanda et al. 2012a; Lima et al. 2010; Cao \& Liang 2011; Liang et al. 2011). In some literature, these two samples have been adopted to test the distance duality relationship, and it turns out that the sample of De Filippis et al. (2005) is more in accordance with no violation of the duality relation (Holanda et al. 2010, 2011, 2012b; Li et al. 2011; Meng et al. 2012).

In this paper, we use the newer and larger sample of galaxy cluster angular size versus redshift data from Bonamante et al. (2006, hereafter B06) to constrain cosmological models that have not been previously considered, and to constrain other cosmological parameters in models that have been previously considered. We show that these constraints are compatible with those derived using other data. We also perform a joint analysis of these angular-size data and SNeIa and BAO measurements and show that including the angular size data in the analysis affects the constraints, although not greatly so as the angular-size data do not yet have sufficient statistical weight.

Our paper is organized as follows. In Sect. 2, we present the basic equations of the three dark energy models we study. Constraints from the B06 angular diameter distances of galaxy clusters are derived in Sect. 3. In Sect. 4, the BAO data and the SNeIa measurements are used to constrain the dark energy models. In Sect. 5, we determine joint constraints on the dark energy parameters from different combinations of the data sets. Finally, we summarize our main conclusions in Sect. 6.

\section{Basic equations of the dark energy models}

The Friedmann equation of the $\Lambda \mathrm{CDM}$ model with spatial curvature can be written as

$E^{2}(z ; \boldsymbol{p})=\Omega_{\mathrm{m} 0}(1+z)^{3}+\Omega_{\Lambda}+\left(1-\Omega_{\mathrm{m} 0}-\Omega_{\Lambda}\right)(1+z)^{2}$,

where $z$ is the redshift, $E(z)=H(z) / H_{0}$ is the dimensionless Hubble parameter where $H_{0}$ is the Hubble constant, and the model-parameter set is $\boldsymbol{p}=\left(\Omega_{\mathrm{m} 0}, \Omega_{\Lambda}\right)$ where $\Omega_{\mathrm{m} 0}$ is the nonrelativistic (baryonic and cold dark) matter density parameter and $\Omega_{\Lambda}$ that of the cosmological constant. Throughout, the subscript 0 denotes the value of a quantity today. In this paper, the subscripts $\Lambda, X$, and $\phi$ represent the corresponding quantities of the dark energy component in the $\Lambda$ CDM, XCDM, and $\phi \mathrm{CDM}$ models.

In this work, for computational simplicity, the spatial curvature is set to zero in the XCDM and $\phi \mathrm{CDM}$ cases. The Friedmann equation for the XCDM parametrization is then

$E^{2}(z ; \boldsymbol{p})=\Omega_{\mathrm{m} 0}(1+z)^{3}+\left(1-\Omega_{\mathrm{m} 0}\right)(1+z)^{3\left(1+w_{X}\right)}$,

where the model-parameter set is $\boldsymbol{p}=\left(\Omega_{\mathrm{m} 0}, w_{X}\right)$. 
In the $\phi \mathrm{CDM}$ model, the inverse power-law potential-energy density of the scalar field adopted in this paper is $V(\phi)=$ $\kappa m_{\mathrm{p}}^{2} \phi^{-\alpha}$, where $m_{\mathrm{p}}$ is the Planck mass, and $\alpha$ and $\kappa$ are nonnegative constants (Peebles \& Ratra 1988). In the spatially flat case, the Friedmann equation of the $\phi \mathrm{CDM}$ model is

$H^{2}(z ; \boldsymbol{p})=\frac{8 \pi}{3 m_{\mathrm{p}}^{2}}\left(\rho_{\mathrm{m}}+\rho_{\phi}\right)$

where $H(z)=\dot{a} / a$ is the Hubble parameter, and $a(t)$ is the cosmological scale factor and an overdot denotes a time derivative. The energy densities of the matter and the scalar field are

$\rho_{m}=\frac{m_{\mathrm{p}}^{2}}{6 \pi} a^{-3}$

and

$\rho_{\phi}=\frac{m_{\mathrm{p}}^{2}}{32 \pi}\left(\dot{\phi}^{2}+\kappa m_{\mathrm{p}}^{2} \phi^{-\alpha}\right)$

respectively. According to the definition of the dimensionless density parameter, one has

$\Omega_{\mathrm{m}}(z)=\frac{8 \pi \rho_{m}}{3 m_{\mathrm{p}}^{2} H^{2}}=\frac{\rho_{m}}{\rho_{m}+\rho_{\phi}}$.

The scalar field $\phi$ obeys the differential equation

$\ddot{\phi}+3 \frac{\dot{a}}{a} \dot{\phi}-\frac{\kappa \alpha}{2} m_{\mathrm{p}}^{2} \phi^{-(\alpha+1)}=0$.

Using Eqs. (3) and (7), as well as the initial conditions described in Peebles \& Ratra (1988), one can numerically compute the Hubble parameter $H(z)$. In this case, the model-parameter set is $\boldsymbol{p}=\left(\Omega_{\mathrm{m} 0}, \alpha\right)$.

To use observational data to constrain cosmological models, we need various distance expressions. The coordinate distance is

$r=\frac{c}{a_{0} H_{0} \sqrt{\left|\Omega_{k}\right|}} \operatorname{sinn}\left[\sqrt{\left|\Omega_{k}\right|} \int_{0}^{z} \frac{\mathrm{d} z^{\prime}}{E\left(z^{\prime}\right)}\right]$

where $\Omega_{k}$ is the spatial curvature density parameter and $c$ is the speed of light, and

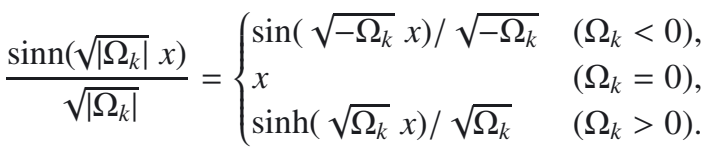

The luminosity distance $d_{\mathrm{L}}$ and the angular diameter distance $d_{\mathrm{A}}$ are simply related to the coordinate distance as

$d_{\mathrm{L}}=\left(a_{0} r\right)(1+z)$

and

$d_{\mathrm{A}}=\left(a_{0} r\right) /(1+z)$
Table 1. Angular diameter distances of galaxy clusters from B06.

\begin{tabular}{lcc}
\hline \hline Cluster & $z$ & $d_{\mathrm{A}}(\mathrm{Mpc})$ \\
\hline Abell 1413 & 0.142 & $780_{-130}^{+180}$ \\
Abell 2204 & 0.152 & $610_{-70}^{+60}$ \\
Abell 2259 & 0.164 & $580_{-250}^{+290}$ \\
Abell 586 & 0.171 & $520_{-120}^{+150}$ \\
Abell 1914 & 0.171 & $440_{-50}^{+40}$ \\
Abell 2218 & 0.176 & $660_{-110}^{+140}$ \\
Abell 665 & 0.182 & $660_{-100}^{+90}$ \\
Abell 1689 & 0.183 & $650_{-90}^{+90}$ \\
Abell 2163 & 0.202 & $520_{-50}^{+40}$ \\
Abell 773 & 0.217 & $980_{-140}^{+170}$ \\
Abell 2261 & 0.224 & $730_{-130}^{+200}$ \\
Abell 2111 & 0.229 & $640_{-170}^{+200}$ \\
Abell 267 & 0.230 & $600_{-90}^{+110}$ \\
RX J2129.7+0005 & 0.235 & $460_{-80}^{+110}$ \\
Abell 1835 & 0.252 & $1070_{-80}^{+20}$ \\
Abell 68 & 0.255 & $630_{-190}^{+160}$ \\
Abell 697 & 0.282 & $880_{-230}^{+300}$ \\
Abell 611 & 0.288 & $780_{-180}^{+180}$ \\
ZW 3146 & 0.291 & $830_{-20}^{+20}$ \\
Abell 1995 & 0.322 & $1190_{-140}^{+150}$ \\
MS 1358.4+6245 & 0.327 & $1130_{-100}^{+90}$ \\
Abell 370 & 0.375 & $1080_{-200}^{+190}$ \\
MACS J2228.5+2036 & 0.412 & $1220_{-230}^{+240}$ \\
RX J1347.5-1145 & 0.451 & $960_{-80}^{+60}$ \\
MACS J2214.9-1359 & 0.483 & $1440_{-230}^{+270}$ \\
MACS J1311.0-0310 & 0.490 & $1380_{-370}^{+470}$ \\
CL 0016+1609 & 0.541 & $1380_{-220}^{+220}$ \\
MACS J1149.5+2223 & 0.544 & $800_{-160}^{+190}$ \\
MACS J1423.8+2404 & 0.545 & $1490_{-30}^{+60}$ \\
MS 0451.6-0305 & 0.550 & $1420_{-230}^{+260}$ \\
MACS J2129.4-0741 & 0.570 & $1330_{-280}^{+370}$ \\
MS 2053.7-0449 & 0.583 & $2480_{-440}^{+10}$ \\
MACS J0647.7+7015 & 0.584 & $770_{-180}^{+210}$ \\
MACS J0744.8+3927 & 0.686 & $1680_{-380}^{+480}$ \\
MS 1137.5+6625 & 0.784 & $2850_{-530}^{+520}$ \\
RX J1716.4+6708 & 0.813 & $1040_{-430}^{+540}$ \\
MS 1054.5-0321 & 0.826 & $1330_{-260}^{+280}$ \\
CL J226.9+3332 & 0.890 & $1080_{-280}^{+420}$ \\
\hline
\end{tabular}

\section{Constraints from the angular-size data}

X-ray observations of the intracluster medium combined with radio observations of the galaxy cluster Sunyaev-Zeldovich effect allow an estimate to be made of the angular diameter distance (ADD) $d_{\mathrm{A}}$ of galaxy clusters. Here we use the 38 ADDs of B06 to constrain cosmological parameters. These data can be found in Tables 1 and 2 of B06. For convenience, we re-collect them in Table 1.

There are three sources of uncertainty in the measurement of $d_{\mathrm{A}}$ : the cluster modeling error $\sigma_{\text {mod }}$, the statistical error $\sigma_{\text {stat }}$, and the systematic error $\sigma_{\text {sys. }}$. The modeling errors are shown in Table 1 and the statistical and systematic errors are presented in Table 3 of B06. In our analysis here, we combine these errors in quadrature. Thus, the total uncertainty $\sigma_{\text {tot }}$ satisfies $\sigma_{\text {tot }}^{2}=\sigma_{\text {mod }}^{2}+\sigma_{\text {stat }}^{2}+\sigma_{\text {sys }}^{2}$. 


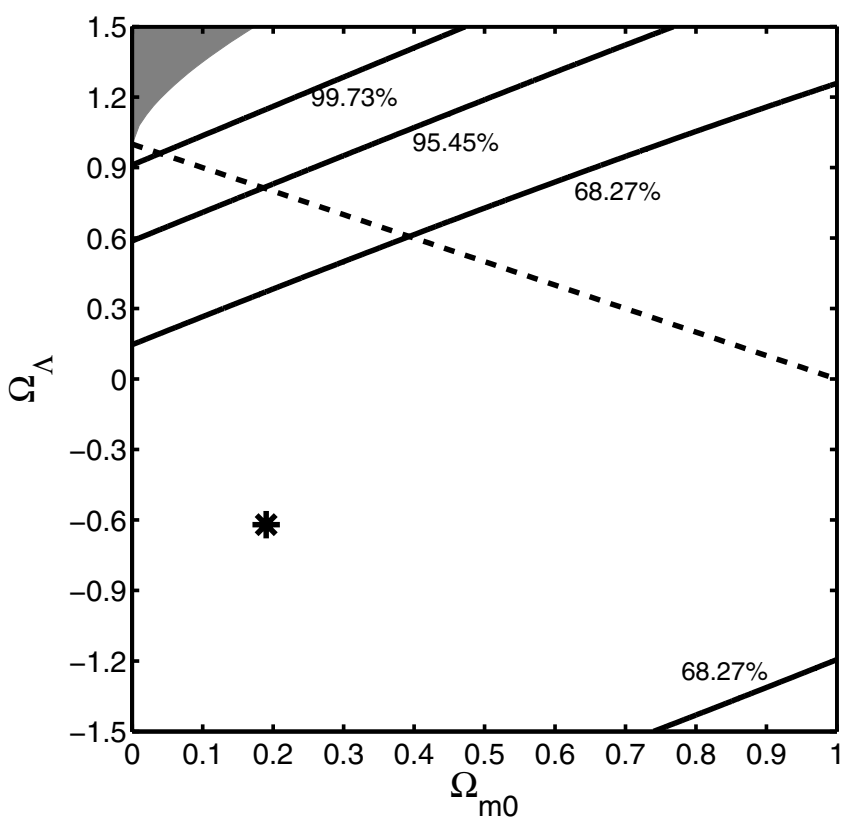

Fig. 1. We display the 1, 2, and $3 \sigma$ constraint contours for the $\Lambda \mathrm{CDM}$ model from the ADD data. The dashed diagonal line corresponds to spatially flat models and the shaded area in the upper lefthand corner is the region for which there is no big bang. The star marks the best-fit pair $\left(\Omega_{\mathrm{m} 0}, \Omega_{\Lambda}\right)=(0.19,-0.62)$ with $\chi_{\min }^{2}=30.1$.

Table 2. Two standard-deviation bounds on cosmological parameters.

\begin{tabular}{lcc}
\hline \hline Model & BAO + SNeIa & ADD + BAO + SNeIa \\
\hline$\Lambda$ CDM & $0.24<\Omega_{\mathrm{m} 0}<0.33$ & $0.24<\Omega_{\mathrm{m} 0}<0.33$ \\
& $0.5<\Omega_{\Lambda}<0.97$ & $0.46<\Omega_{\Lambda}<0.93$ \\
\hline $\mathrm{XCDM}$ & $0.24<\Omega_{\mathrm{m} 0}<0.33$ & $0.24<\Omega_{\mathrm{m} 0}<0.33$ \\
& $-1.30<\omega_{X}<-0.80$ & $-1.25<\omega_{X}<-0.77$ \\
\hline$\phi \mathrm{CDM}$ & $0.24<\Omega_{\mathrm{m} 0}<0.33$ & $0.24<\Omega_{\mathrm{m} 0}<0.33$ \\
& $0<\alpha<0.73$ & $0.01<\alpha<0.89$ \\
\hline
\end{tabular}

We constrain cosmological parameters by minimizing $\chi_{\mathrm{ADD}}^{2}$

$\chi_{\mathrm{ADD}}^{2}\left(H_{0}, \boldsymbol{p}\right)=\sum_{i=1}^{38} \frac{\left[d_{\mathrm{A}}^{\mathrm{th}}\left(z_{i} ; H_{0}, \boldsymbol{p}\right)-d_{\mathrm{A}}^{\mathrm{obs}}\left(z_{i}\right)\right]^{2}}{\sigma_{\mathrm{tot}, i}^{2}}$.

Here $z_{i}$ is the redshift of the observed galaxy cluster, $d_{\mathrm{A}}^{\text {th }}$ is the predicted value of the ADD in the cosmological model under consideration and $d_{\mathrm{A}}^{\mathrm{obs}}$ is the measured value. From $\chi_{\mathrm{ADD}}^{2}\left(H_{0}, \boldsymbol{p}\right)$, we compute the likelihood function $L\left(H_{0}, \boldsymbol{p}\right)$. We then treat $H_{0}$ as a nuisance parameter and marginalize over it using a Gaussian prior with $H_{0}=68 \pm 3.5 \mathrm{~km} \mathrm{~s}^{-1} \mathrm{Mpc}^{-1}$ (Chen et al. 2003) to get a likelihood function $L(\boldsymbol{p})$ that is a function only of the cosmological parameters of interest. The best-fit parameter values $p *$ are those that maximize the likelihood function and the 1,2 , and $3 \sigma$ constraint contours are the set of cosmological parameters (centered on $p *$ ) that enclose the $68.27 \%, 95.45 \%$ and $99.73 \%$ confidence levels, respectively, of the probability under the likelihood function.

Figures 1-3 show the constraints from the ADD data on the three dark energy models we consider. Comparing these results to those shown in Figs. 1-3 of Chen \& Ratra (2003a), which were derived using the compact radio source angular-size data of Gurvits et al. (1999), and to Figs. 1, 2 of Podariu et al. (2003), derived using the FRIIb radio galaxy angular-size data from

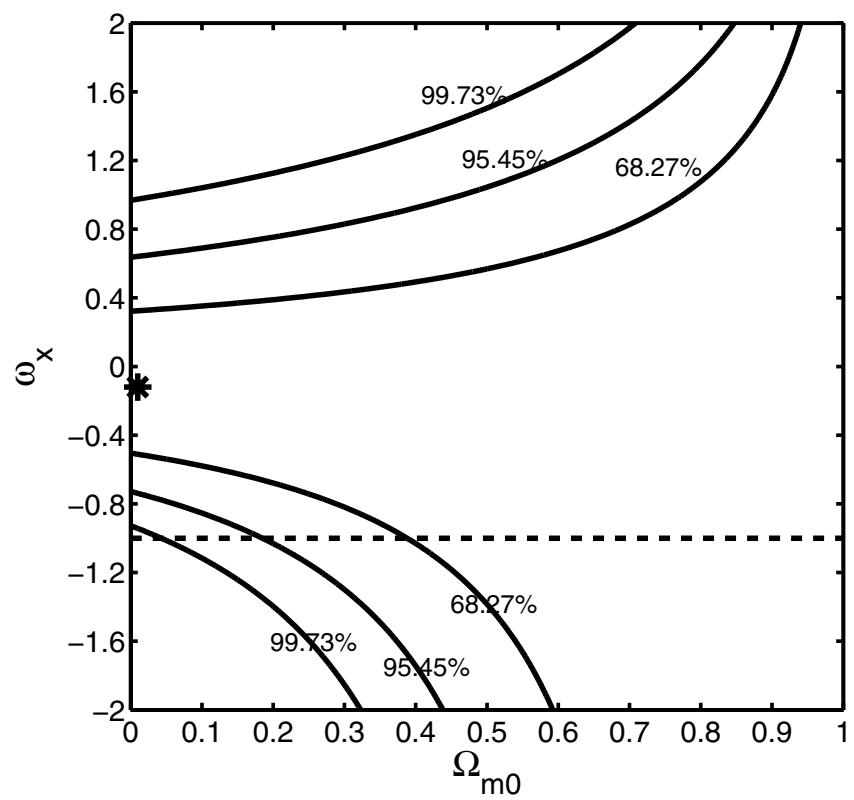

Fig. 2. We show the 1,2 , and $3 \sigma$ constraint contours for the XCDM parametrization from the ADD data. The dashed horizontal line at $\omega_{X}=-1$ corresponds to spatially flat $\Lambda \mathrm{CDM}$ models. The star marks the best-fit pair $\left(\Omega_{\mathrm{m} 0}, w_{X}\right)=(0.01,-0.12)$ with $\chi_{\min }^{2}=30.2$.

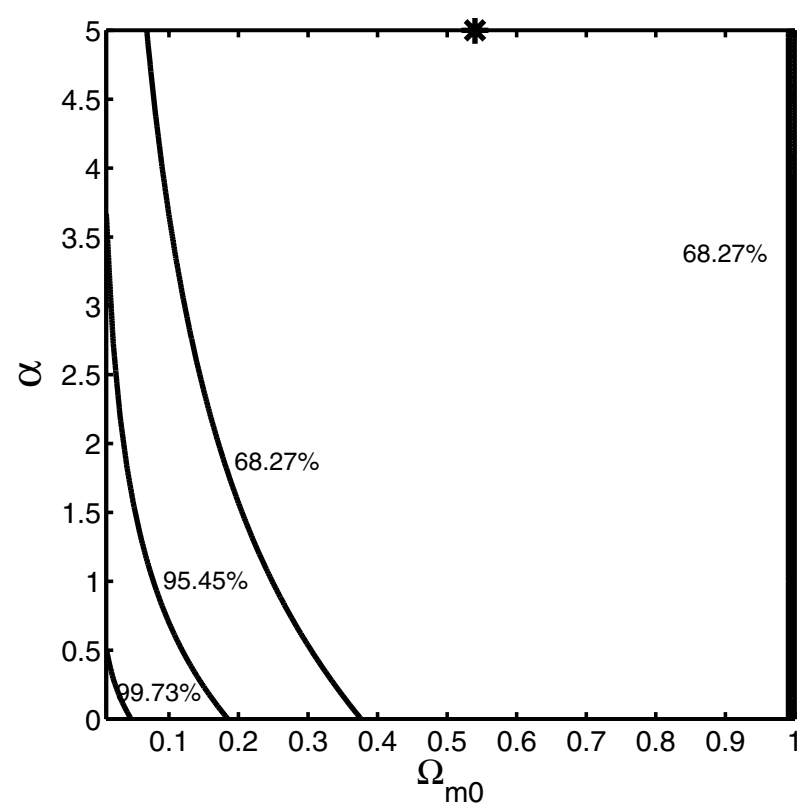

Fig. 3. We perform the 1, 2, and $3 \sigma$ constraint contours for the $\phi \mathrm{CDM}$ model from the ADD data. The horizontal axis at $\alpha=0$ corresponds to spatially flat $\Lambda \mathrm{CDM}$ models. The star marks the best-fit pair $\left(\Omega_{\mathrm{m} 0}, \alpha\right)=(0.54,5)$ with $\chi_{\min }^{2}=37.3$.

Guerra et al. (2000), we see that the B06 galaxy cluster angularsize data provide approximately comparable constraints on cosmological parameters as those derived from the two earlier angular-size data sets. These ADD constraints are comparable to those from gamma-ray burst data (Samushia \& Ratra 2010, Figs. 1-3 and 7-9), as well as those from Hubble parameter measurements (Samushia et al. 2007, Figs. 1-3).

Current ADD data constraints are clearly not very restrictive, although it is encouraging that the ADD constraints on these 


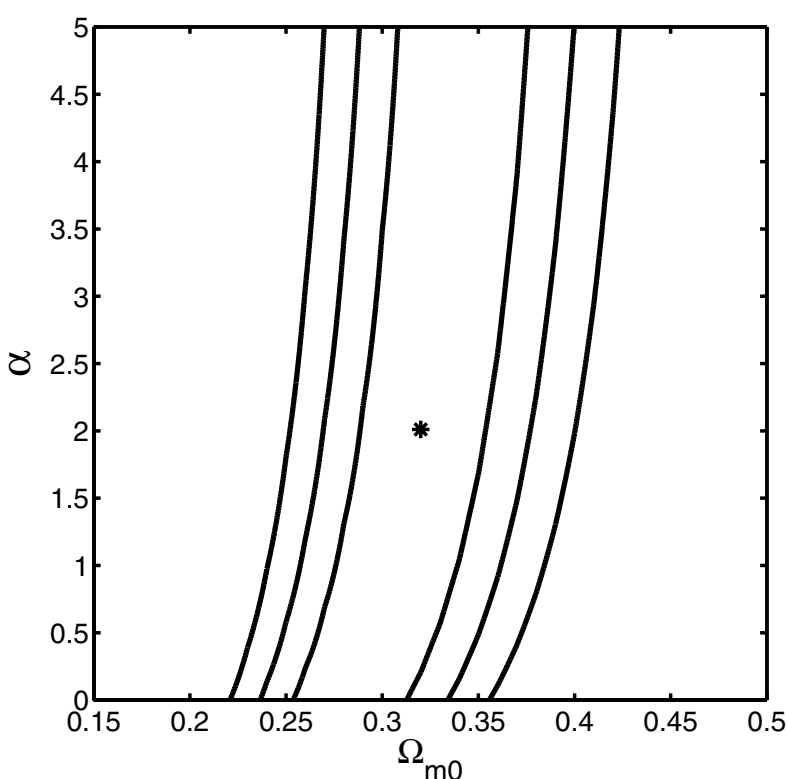

Fig. 4. We show the 1,2 , and $3 \sigma$ constraint contours for the $\phi \mathrm{CDM}$ model derived from the BAO data. The horizontal axis at $\alpha=0$ corresponds to spatially flat $\Lambda \mathrm{CDM}$ models. The star marks the best-fit pair $\left(\Omega_{\mathrm{m} 0}, \alpha\right)=(0.32,2.01)$ with $\chi_{\min }^{2}=0.169$.

dark energy models do not disfavor the regions of parameter space that are favored by other data. More importantly, we anticipate that ADD data to be acquired in the near future will provide significantly tighter constraints on cosmological parameters.

\section{Constraints from BAO and SNela data}

The BAO peak length scale can be used as a standard ruler to constrain cosmological parameters. Here we use the BAO data of Percival et al. (2010) to constrain the parameters of the $\Lambda \mathrm{CDM}$ and $\phi \mathrm{CDM}$ models and the XCDM parametrization.

Percival et al. (2010) measure the position of the BAO peak from the SDSS DR7 and 2dFGRS data, determining $r_{\mathrm{s}}\left(z_{\mathrm{d}}\right) / D_{V}(z=0.275)=0.1390 \pm 0.0037$, where $r_{\mathrm{s}}\left(z_{\mathrm{d}}\right)$ is the comoving sound horizon at the baryon drag epoch, and $D_{V}(z) \equiv$ $\left[(1+z)^{2} d_{\mathrm{A}}^{2} c z / H(z)\right]^{1 / 3}$. By using $\Omega_{\mathrm{m} 0} h^{2}=0.1326 \pm 0.0063$ and $\Omega_{\mathrm{b} 0} h^{2}=0.02273 \pm 0.00061$ (where $\Omega_{\mathrm{b} 0}$ is the current value of the baryonic mass density parameter and $h$ is the Hubble constant in units of $100 \mathrm{~km} \mathrm{~s}^{-1} \mathrm{Mpc}^{-1}$ ) from WMAP5 (Komatsu et al. 2009), one can get

$D_{V}(0.275)=(1104 \pm 30)\left(\frac{\Omega_{\mathrm{b} 0} h^{2}}{0.02273}\right)^{-0.134}\left(\frac{\Omega_{\mathrm{m} 0} h^{2}}{0.1326}\right)^{-0.255} \mathrm{Mpc}$

as shown in Eq. (13) of Percival et al. (2010). The error in $\Omega_{\mathrm{b} 0} h^{2}$ is ignored in this work, as the WMAP5 data constrains $\Omega_{\mathrm{b} 0} h^{2}$ to within $0.5 \%$.

Our results for the $\Lambda \mathrm{CDM}$ model and the XCDM parametrization agree very well with the Percival et al. (2010) results shown in their Fig. 5. Our results for the $\phi$ CDM model are shown in Fig. 4. The BAO data constrains $\Omega_{\mathrm{m} 0}$ significantly, leaving $\Omega_{\Lambda}, w_{X}$, and $\alpha$ almost unconstrained (see, e.g., Samushia $\&$ Ratra 2009a). The results obtained from the BAO data of Percival et al. (2010) are most directly comparable to those

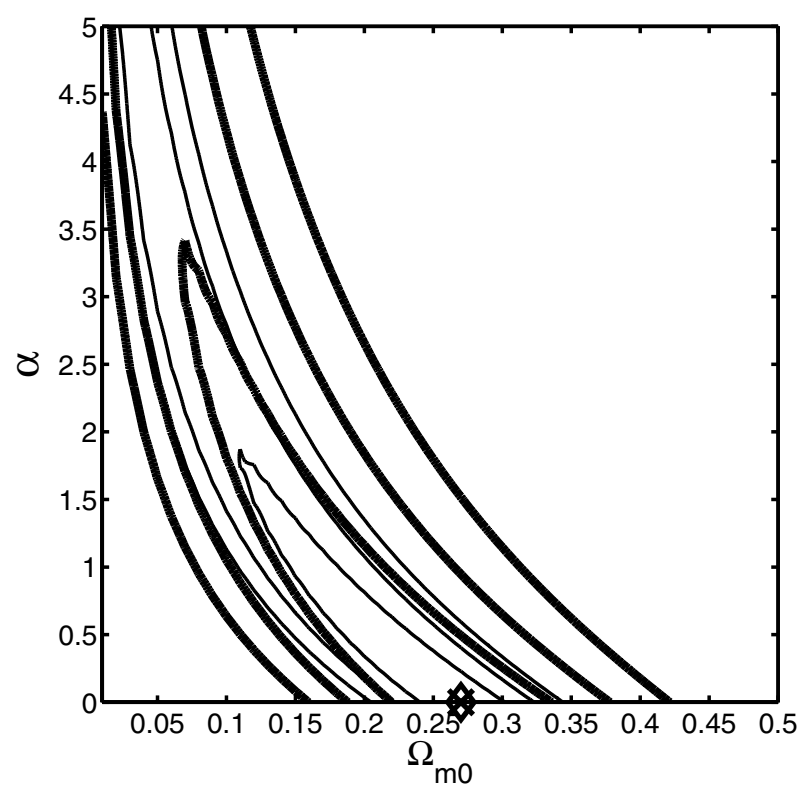

Fig. 5. We display the 1, 2, and $3 \sigma$ constraint contours for the $\phi \mathrm{CDM}$ model from the SNeIa data. The horizontal axis at $\alpha=0$ corresponds to spatially flat $\Lambda \mathrm{CDM}$ models. Thin solid lines (best fit at $\Omega_{\mathrm{m} 0}=0.27$ and $\alpha=0.0$ with $\chi_{\min }^{2}=543$, marked by " $\times$ ") ) exclude systematic errors, while thick solid lines (best fit at $\Omega_{\mathrm{m} 0}=0.27$ and $\alpha=0.0$ with $\chi_{\min }^{2}=531$, marked by “ $\diamond$ ") account for systematics.

derived from the earlier BAO data of Eisenstein et al. (2005). Comparing to the constraints shown in Figs. 2-4 of Samushia \& Ratra (2009a), one sees that the Percival et al. (2010) data lead to slightly more restrictive constraints than the Eisenstein et al. (2005) data.

Type Ia supernovae are standardizable candles that can be used to constrain cosmological parameters. Here we use the Union2 compliation of 557 SNeIa (covering a redshift range $0.015 \leq z \leq 1.4$ ) of Amanullah et al. (2010) to constrain parameters of the $\Lambda \mathrm{CDM}$ and $\phi \mathrm{CDM}$ models and the XCDM parametrization.

Cosmological constraints from SNeIa data are obtained by using the distance modulus $\mu(z)$. The theoretical (predicted) distance modulus is

$\mu_{\mathrm{th}}\left(z ; \boldsymbol{p}, \mu_{0}\right)=5 \log _{10}\left[D_{\mathrm{L}}(z ; \boldsymbol{p})\right]+\mu_{0}$,

where $\mu_{0}=42.38-5 \log _{10} h$ and the Hubble-free luminosity distance is given by

$D_{\mathrm{L}}(z ; \boldsymbol{p})=\frac{H_{0}}{c} d_{\mathrm{L}}=(1+z) \int_{0}^{z} \frac{\mathrm{d} z^{\prime}}{E\left(z^{\prime} ; \boldsymbol{p}\right)}$.

The best-fit values of cosmological model parameters can be determined by minimizing the $\chi^{2}$ function

$\chi_{S N}^{2}\left(\boldsymbol{p}, \mu_{0}\right)=\sum_{i=1}^{557} \frac{\left[\mu_{\mathrm{th}, i}\left(z_{i} ; \boldsymbol{p}, \mu_{0}\right)-\mu_{\mathrm{obs}, i}\left(z_{i}\right)\right]}{\sigma_{\mu_{i}}^{2}}$,

where $\mu_{\mathrm{obs}, i}\left(z_{i}\right)$ is the distance modulus obtained from observations and $\sigma_{\mu_{i}}$ is the total uncertainty in the SNeIa data. The zeropoint $\mu_{0}$ is treated as a nuisance parameter and marginalized over analytically (Di Pietro \& Claeskens 2003; Perivolaropoulos 2005; Nesseris \& Perivolaropoulos 2005). The covariance matrix with or without systematic errors can be found on the web ${ }^{1}$.

1 http://supernova.lbl.gov/Union 


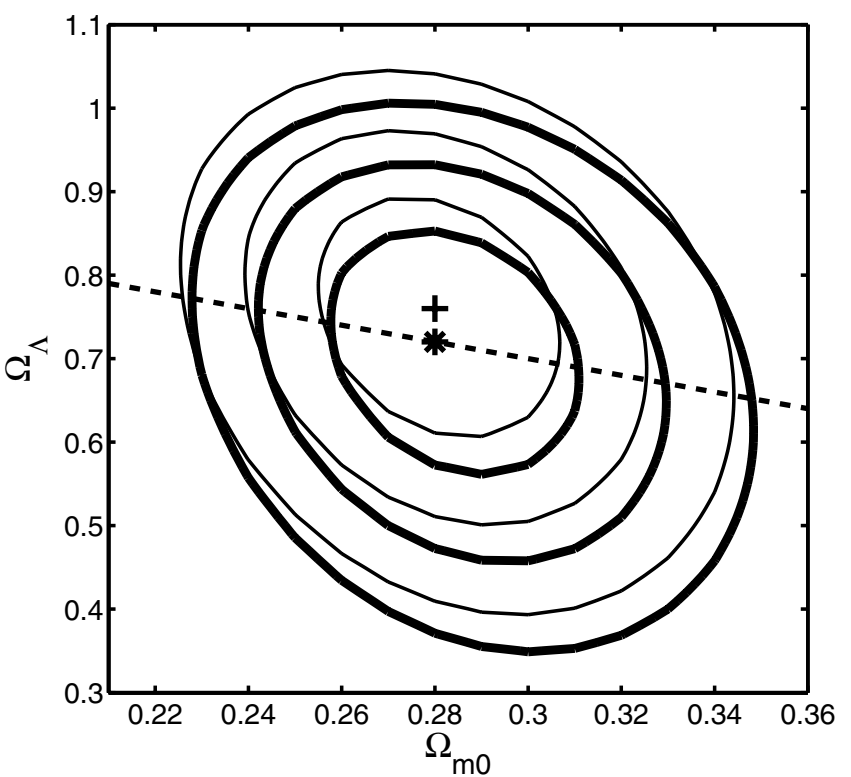

Fig. 6. Thick (thin) solid lines are 1,2, and $3 \sigma$ constraint contours for the $\Lambda$ CDM model from a joint analysis of the BAO and SNeIa (with systematic errors) data, with (and without) the ADD data. The cross ("+") marks the best-fit point determined from the joint sample without the ADD data at $\Omega_{\mathrm{m} 0}=0.28$ and $\Omega_{\Lambda}=0.76$ with $\chi_{\min }^{2}=531$. The star ("**) marks the best-fit point determined from the joint sample with the ADD data at $\Omega_{\mathrm{m} 0}=0.28$ and $\Omega_{\Lambda}=0.72$ with $\chi_{\min }^{2}=565$. The dashed sloping line corresponds to spatially flat models.

Our results for the $\Lambda$ CDM model and the XCDM parametrization agree very well with the Amanullah et al. (2010) results shown in their Figs. 10 and 11. The constraints on $\phi$ CDM model parameters from these data are shown in Fig. 5. Comparing to Fig. 4 of Samushia \& Ratra (2009b), we can see that the constraints of the Amanullah et al. (2010) data with systematic errors are approximately comparable to those of the earlier Kowalski et al. (2008) data for 307 SNeIa without consideration of their systematic errors. As for the BAO data, the SNeIa data constraints are also fairly one-dimensional, tightly constraining one combination of the cosmological parameters, while only weakly constraining the "orthogonal" combination.

\section{Joint constraints}

Figures 6-8 show the constraints provided on the cosmological parameters of both the $\Lambda \mathrm{CDM}$ and $\phi \mathrm{CDM}$ models and the XCDM parametrization by a joint analysis of the BAO and SNeIa data, as well as by a joint analysis of the BAO, SNeIa, and ADD data. With the inclusion of systematic errors in the analysis of the SNeIa data of Amanullah et al. (2010), the new joint BAO and SNeIa constraints (thin solid contours in Figs. 6-8) are similar to the earlier ones shown in Figs. 4-6 of Samushia \& Ratra (2010), which made use of the smaller SNeIa data set of Kowalski et al. (2008) that did not include systematic errors.

Figures 9-11 display the one-dimensional marginalized distribution probabilities of the cosmological parameters for the three cosmological models considered in this work, derived from a joint analysis of the BAO and SNeIa data, as well as from a joint analysis of the BAO, SNeIa, and ADD data. The marginalized $2 \sigma$ intervals of the cosmological parameters are presented in Table 2.

The combination of BAO and SNeIa data gives tight constraints on the cosmological parameters. Adding the currently

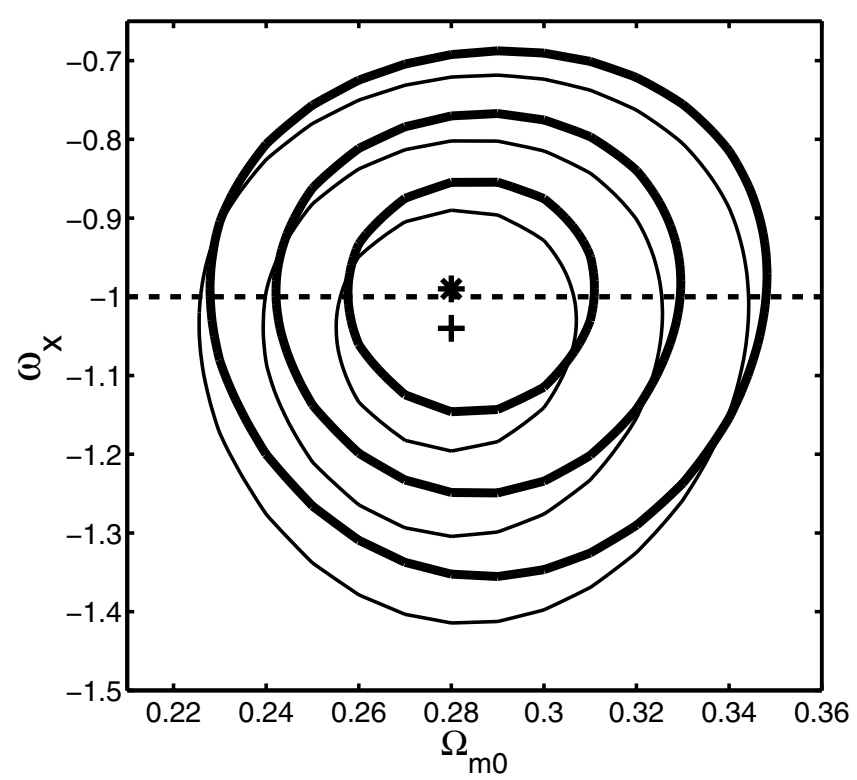

Fig. 7. Thick (thin) solid lines are 1, 2, and $3 \sigma$ constraint contours for the XCDM parametrization from a joint analysis of the BAO and SNeIa (with systematic errors) data, with (and without) the ADD data. The cross ("+") marks the best-fit point determined from the joint sample without the ADD data at $\Omega_{\mathrm{m} 0}=0.28$ and $\omega_{X}=-1.04$ with $\chi_{\min }^{2}=531$. The star ("*") marks the best-fit point determined from the joint sample with the ADD data at $\Omega_{\mathrm{m} 0}=0.28$ and $\omega_{X}=-0.99$ with $\chi_{\min }^{2}=565$. The dashed horizontal line at $\omega_{X}=-1$ corresponds to spatially flat $\Lambda$ CDM models.

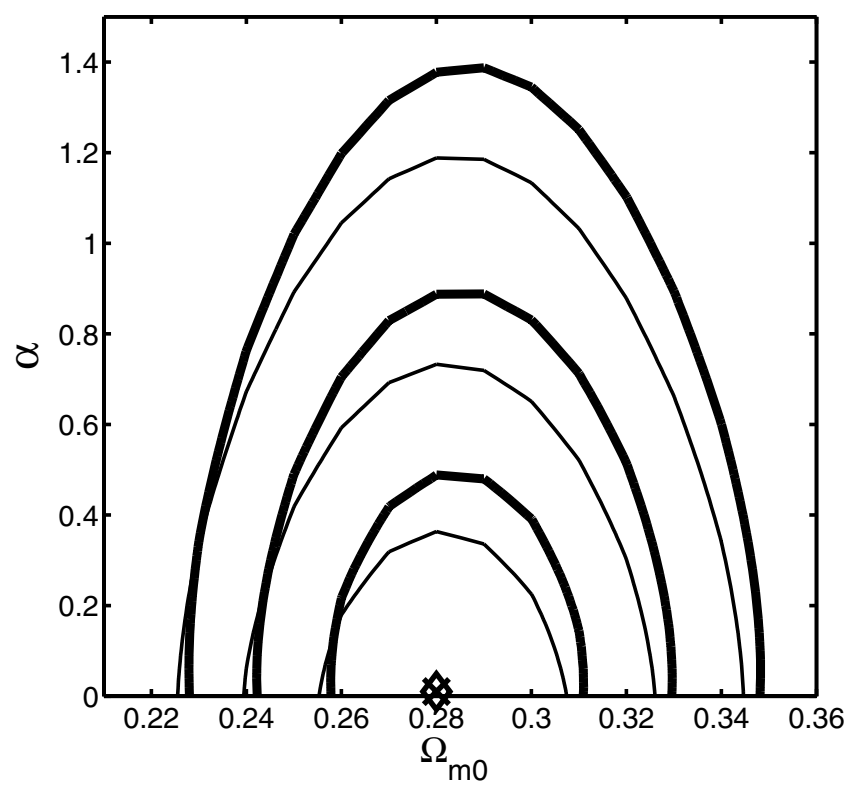

Fig. 8. Thick (thin) solid lines are 1, 2, and $3 \sigma$ constraint contours for the $\phi C D M$ model from a joint analysis of the BAO and SNeIa (with systematic errors) data, with (and without) the ADD data. The cross (" $X$ ") marks the best-fit point determined from the joint sample without the ADD data at $\Omega_{\mathrm{m} 0}=0.28$ and $\alpha=0$ with $\chi_{\min }^{2}=531$. The diamond (" $\diamond ")$ marks the best-fit point determined from the joint sample with the ADD data at $\Omega_{\mathrm{m} 0}=0.28$ and $\alpha=0.01$ with $\chi_{\min }^{2}=572$. The $\alpha=0$ horizontal axis corresponds to spatially flat $\Lambda \mathrm{CDM}$ models.

available galaxy-cluster ADD data to this combination does shift the constraint contours, although the effect is not large. Current ADD data do not have enough statistical weight to significantly affect the combined BAO and SNeIa results. The ADD data have 

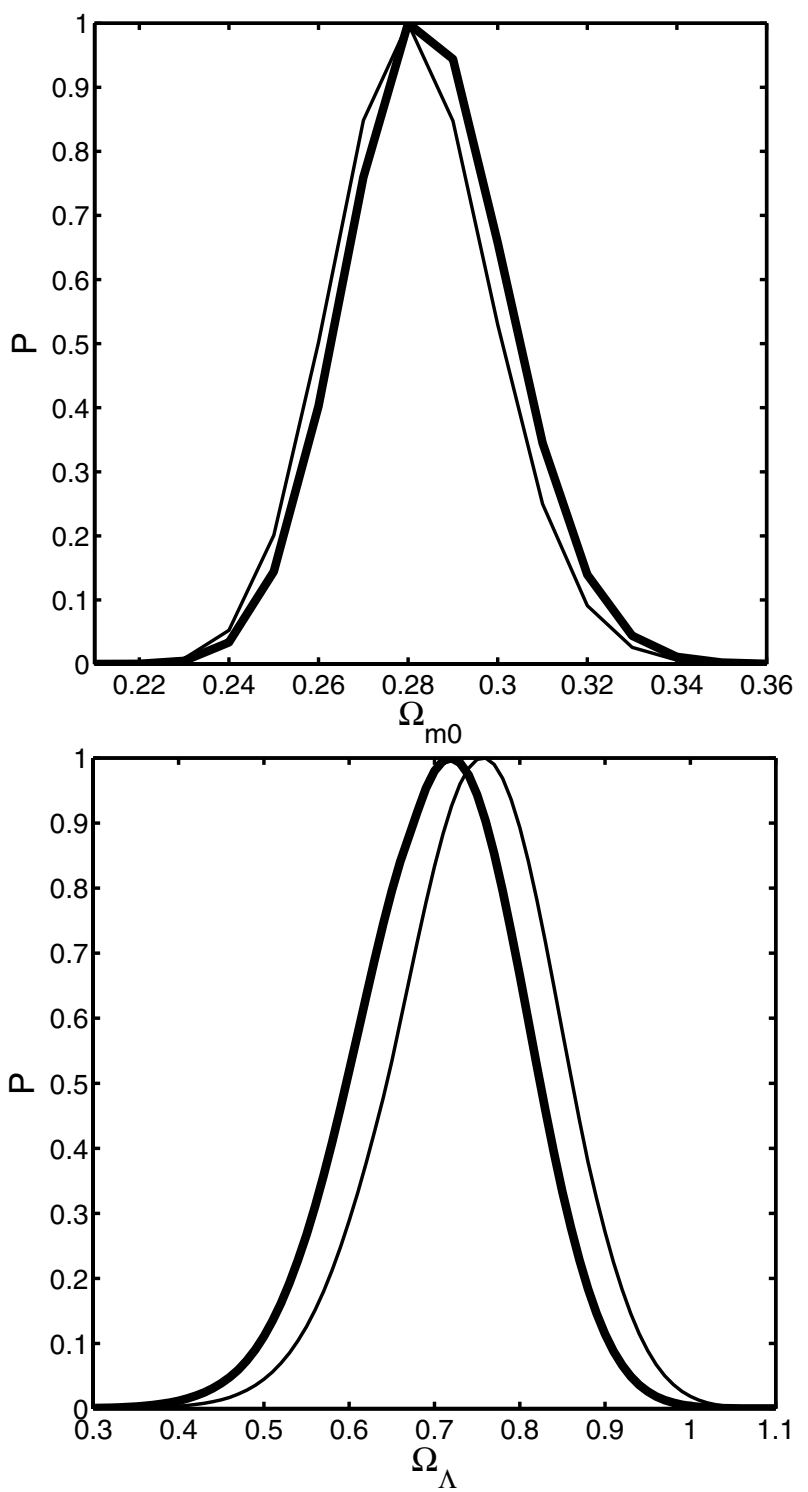

Fig. 9. One-dimensional marginalized distribution probabilities of the cosmological parameters for the LCDM model. Thick (thin) lines are the results from a joint analysis of the BAO and SNeIa (with systematic errors) data, with (and without) the ADD data.

approximately the same weight as currently available gammaray burst luminosity measurements (Samushia \& Ratra 2010, Figs. 4-6 and 10-12).

The observational data considered here are clearly very consistent with the predictions of a spatially-flat cosmological model with an energy budget dominated by a time-independent cosmological constant. However, the data do not rule out timeevolving dark energy, although they do require that it not vary rapidly.

\section{Conclusion}

We have shown that the galaxy-cluster angular size versus redshift data of B06 can also be used to constrain dark energy model cosmological parameters. The resulting constraints are compatible with those derived from other sets of current data, thus strengthening support for the current "standard" cosmological model. The ADD constraints are approximately as restrictive as those that follow from currently available gamma-ray burst
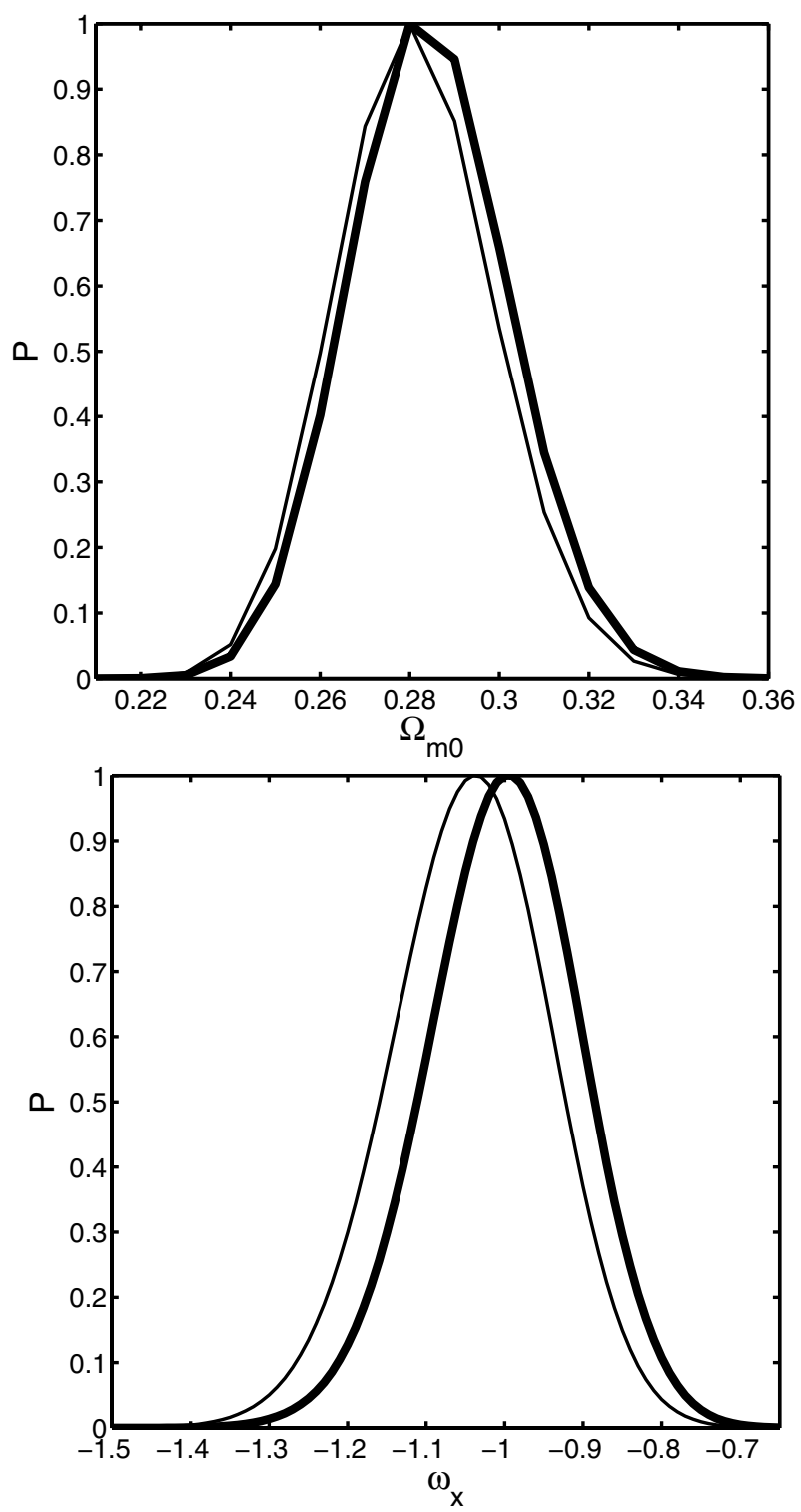

Fig. 10. One-dimensional marginalized distribution probabilities of the cosmological parameters for the XCDM parametrization. Thick (thin) lines are the results from a joint analysis of the BAO and SNeIa (with systematic errors) data, with (and without) the ADD data.

luminosity data, strong gravitational-lensing measurements, or lookback time (or Hubble parameter) observations. They are, however, much less restrictive than those that follow from a combined analysis of BAO peak length scale and SNeIa apparent-magnitude data.

The spatially flat $\Lambda \mathrm{CDM}$ model, currently dominated by a constant cosmological constant, provides a good fit to the data that we have studied here. However, these data are not inconsistent with a time-evolving dark energy.

We anticipate that angular-size data to be acquired in the near future will provide significantly tighter constraints on cosmological parameters. In conjunction with other observations, these angular-size data will prove very useful in pinning down parameter values of the "standard" cosmological model.

Acknowledgements. Yun Chen thanks Zong-Hong Zhu and Lado Samushia for their generous and helpful advice. Y.C. was supported by the Ministry of Science and Technology national basic science program (project 973) under grant No. 2012CB821804. BR was supported by DOE grant DEFG03-99EP41093. 

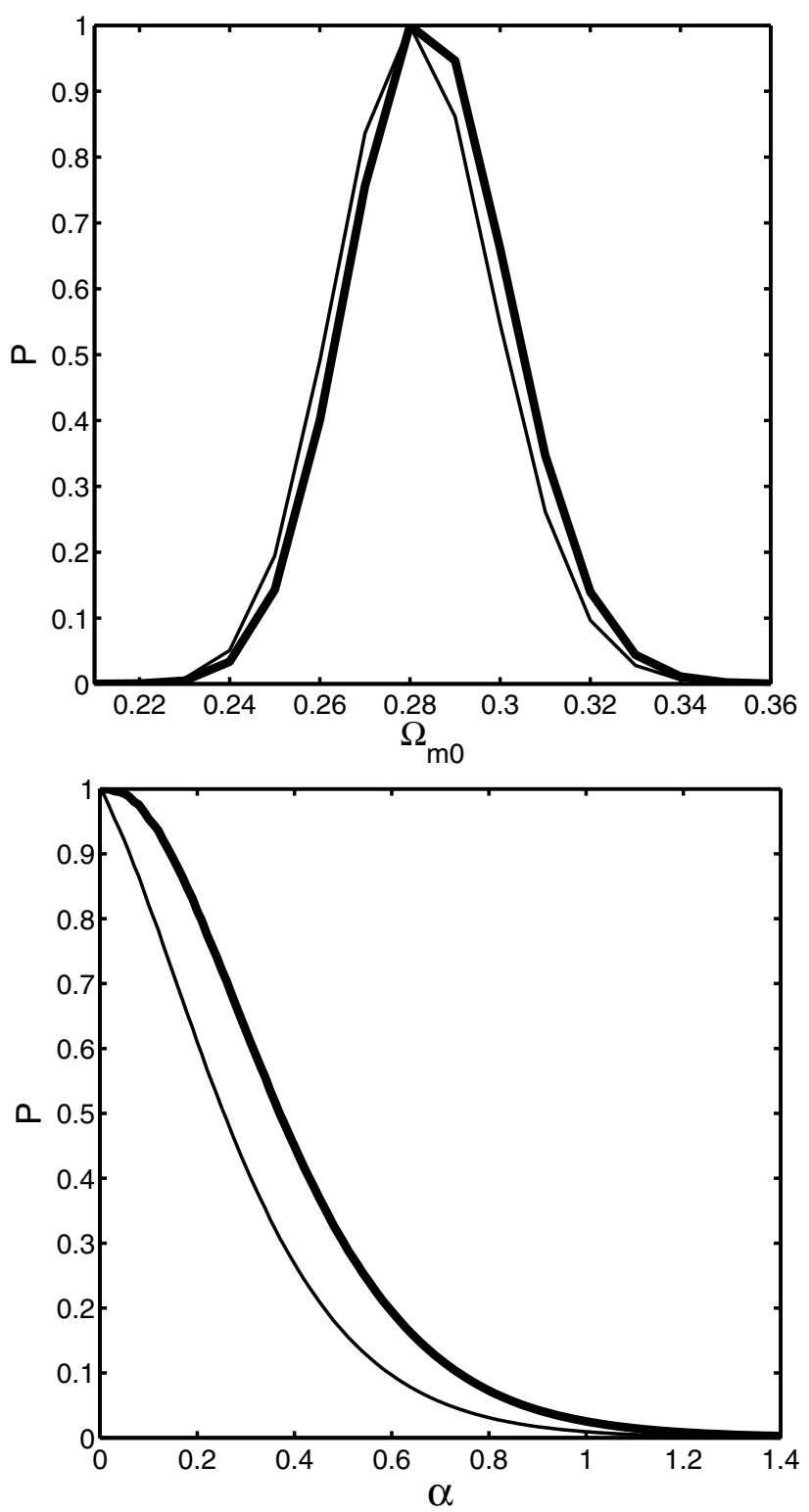

Fig. 11. One-dimensional marginalized distribution probabilities of the cosmological parameters for the $\phi \mathrm{CDM}$ model. Thick (thin) lines are the results of a joint analysis of the BAO and SNeIa (with systematic errors) data, with (and without) the ADD data.

\section{References}

Allen, S. W., Rapetti, D. A., Schmidt, R. W., et al. 2008, MNRAS, 383, 879 Amanullah, R., Lidman, C., Rubin, D., et al. 2010, ApJ, 716, 712 Baldi, M. 2011, MNRAS, 411, 1077

Bartelmann, M. 2010, Rev. Mod. Phys., 82, 331

Basilakos, S., Plionis, M., \& Solà, J. 2010, Phys. Rev. D, 82, 083512

Bonamente, M., Chapman, S. C., Ibata, R. A., et al. 2006, ApJ, 647, 25 (B06) Brax, P. 2009 [arXiv: 0912. 3610]

Cai, Y.-F., Saridakis, E. N., Setare, M. R., et al. 2010, Phys. Rept., 493, 1

Caldwell, R. R., \& Kamionkowski, M. 2009, Ann. Rev. Nucl. Part. Sci., 59, 397 Cao, S., \& Liang, N. 2011, RA\&A, 11, 1199

Capozziello, S., Cardone, V. F., Funaro, M., et al. 2004, Phys. Rev. D, 70, 123501

Chae, K.-H., Biggs, A. D., Blandford, R. D., et al. 2002, Phys. Rev. Lett., 89, 151301

Chae, K.-H., Chen, G., Ratra, B., et al. 2004, ApJ, 607, L71

Chen, G., \& Ratra, B. 2003a, ApJ, 582, 586

Chen, G., \& Ratra, B. 2003b, PASP, 115, 1143

Chen, G., Gott, J. R., \& Ratra, B. 2003, PASP, 115, 1269

Courtin, J., Rasera, Y., Alimi, J.-M., et al. 2011, MNRAS, 410, 1911

Cunha, J. V., Marassi, L., \& Lima, J. A. S. 2007, MNRAS, 379, L1

Dantas, M. A., Alcaniz, J. S., Mania, D., et al. 2011, Phys. Lett. B, 699, 239
Davis, T. M., Mörtsell, E., Sollerman, J., et al. 2007, ApJ, 666, 716

De Bernardis, F., Giusarma, E., \& Melchiorri, A. 2006, Int. J. Mod. Phys. D, 15, 759

De Filippis, E., Sereno, M., Bautz, M. W., et al. 2005, ApJ, 625, 108

Di Pietro, E., \& Claeskens, J.-F. 2003, MNRAS, 341, 1299

Dutta, S., \& Scherrer, R. J. 2010, Phys. Rev. D, 82, 043526

Eisenstein, D. J., Zehavi, I., Hogg, D. W., et al. 2005, ApJ, 633, 560

Ettori, S., Morandi, A., Tozzi, P., et al. 2009, A\&A, 501, 61

Fernandez-Martinez, E., \& Verde, L. 2008, J. Cosmol. Astropart. Phys., 0808, 023

Frieman, J. A. 2008, AIP Conf. Proc., 1057, 87

Gaztañaga, E., Cabré, A., \& Hui, L. 2009, MNRAS, 399, 1663

Guerra, E. J., Daly, R. A., \& Wan, L. 2000, ApJ, 544, 659

Gurvits, L. I., Kellermann, K. I., \& Frey, S. 1999, A\&A, 342, 378

Holanda, R. F. L., Lima, J. A. S., \& Ribeiro, M. B. 2010, ApJ, 722, L233

Holanda, R. F. L., Lima, J. A. S., \& Ribeiro M. B. 2011, A\&A, 528, L14

Holanda, R. F. L., Cunha, J. V., \& Lima, J. A. S. 2012a, Gen. Relativ. Gravit., 44, 501

Holanda, R. F. L., Lima, J. A. S., \& Ribeiro, M. B. 2012b, A\&A, 538, A131

Holsclaw, T., Alam, U., Sansó, B., et al. 2010, Phys. Rev. Lett., 105, 241302

Honorez, L. L., Reid, B. A., Mena, O., Verde, L., \& Jimenez, R. 2010, J. Cosmol. Astropart. Phys., 1009, 029

Jamil, M., \& Saridakis, E. N. 2010, J. Cosmol. Astropart. Phys., 1007, 028

Jassal, H. K., Bagla, J. S., \& Padmanabhan, T. 2010, MNRAS, 405, 2639

Komatsu, E., Dunkley, J., Nolta, M. R., et al. 2009, ApJS, 180, 330

Komatsu, E., Smith, K. M., Dunkley, J., et al. 2011, ApJS, 192, 18

Kowalski, M., Rubin, D., Aldering, G., et al. 2008, ApJ, 686, 749

Lee, S., \& Ng, K.-W. 2007, Phys. Rev. D, 76, 043518

Lepe, S., \& Peña, F. 2010, Eur. Phys. J. C, 69, 575

Li, Z., Wu, P., \& Yu, H. 2011, ApJ, 729, L14

Liang, N., \& Zhang, L. N. 2008, AIP Conf. Proc., 1065, 367

Liang, N., Cao, S., Liao, K., \& Zhu, Z.-H. 2011 [arXiv: 1104 .2497]

Lima, J. A. S., \& Alcaniz, J. S. 2000, A\&A, 357, 393

Lima, J. A. S., \& Alcaniz, J. S. 2002, ApJ, 566, 15

Lima, J. A. S., Holanda, R. F. L., \& Cunha, J. V. 2010, AIP Conf. Proc., 1241, 224 Liu, D.-J. 2010, Phys. Rev. D, 82, 063523

Maggiore, M. 2011, Phys. Rev. D, 83, 063514

Meng, X.-L., Zhang, T.-J., \& Zhan, H. 2012, ApJ, 745, 98

Nesseris, S., \& Perivolaropoulos, L. 2005, Phys. Rev. D, 72, 123519

Peebles, P. J. E. 1984, ApJ, 284, 439

Peebles, P. J. E., \& Ratra, B. 1988, ApJ, 325, L17

Peebles, P. J. E., \& Ratra, B. 2003, Rev. Mod. Phys., 75, 559

Perivolaropoulos, L. 2010, J. Phys. Conf. Ser., 222, 012024

Percival, W. J., Cole, S., Eisenstein, D. J., et al. 2007, MNRAS, 381, 1053

Percival, W. J., Reid, B. A., Eisenstein, D. J., et al. 2010, MNRAS, 401, 2148

Perivolaropoulos, L. 2005, Phys. Rev. D, 71, 063503

Perlmutter, S., Aldering, G., Goldhaber, G., et al. 1999, ApJ, 517, 565

Podariu, S., Nugent, P., \& Ratra, B. 2001a, ApJ, 553, 39

Podariu, S., Ohyama, Y., Murayama, T., et al. 2001b, ApJ, 559, 9

Podariu, S., Daly, R. A., Mory, M. P., \& Ratra, B. 2003, ApJ, 584, 577

Ratra, B., \& Peebles, P. J. E. 1988, Phys. Rev. D, 37, 3406

Ratra, B. 1991, Phys. Rev. D, 43, 3802

Ratra, B., Stompor, R., Ganga, K., et al. 1999, ApJ, 517, 549

Ratra, B., \& Vogeley, M. S. 2008, PASP, 120, 235

Riess, A. G., Filippenko, A. V., Challis, P., et al. 1998, AJ, 116, 1009

Sami, M. 2009, Curr. Sci., 97, 887

Samushia, L., \& Ratra, B. 2006, ApJ, 650, L5

Samushia, L., \& Ratra, B. 2008, ApJ, 680, L1

Samushia, L., \& Ratra, B. 2009a, ApJ, 701, 1373

Samushia, L., \& Ratra, B. 2009b, ApJ, 703, 1904

Samushia, L., \& Ratra, B. 2010, ApJ, 714, 1347

Samushia, L., Chen, G., \& Ratra, B. 2007 [arXiv : 0706. 1963]

Samushia, L., Dev, A., Jain, D., et al. 2010, Phys. Lett. B, 693, 509

Samushia, L., Percival, W. J., Guzzo, L., et al. 2011, MNRAS, 410, 1993

Santos, R. C., \& Lima, J. A. S. 2008, Phys. Rev. D, 77, 083505

Schaefer, B. E. 2007, ApJ, 660, 16

Shafieloo, A., Sahni, V., \& Starobinsky, A. A. 2009, Phys. Rev. D, 80, 101301 Shao, S.-H., \& Chen, P. 2010, Phys. Rev. D, 82, 126012

Simon, J., Verde, L., \& Jimenez, R. 2005, Phys. Rev. D, 71, 123001

Sloth, M. S. 2010, Int. J. Mod. Phys. D, 19, 2259

Spergel, D. N., Verde, L., Peiris, H. V., et al. 2003, ApJS, 148, 175

Wang, Y. 2008, Phys. Rev. D, 78, 123532

Wang, Y. 2010, Mod. Phys. Lett. A, 25, 3093

Wang, Y., Percival, W., Cimatti, A., et al. 2010, MNRAS, 409, 737

Wei, H. 2011, Phys. Lett. B, 695, 307

Wilson, K. M., Chen, G., \& Ratra, B. 2006, Mod. Phys. Lett. A, 21, 2197

Yang, R.-J. \& Zhang, S. N. 2010, MNRAS, 407, 1835

Yashar, M., Bozek, B., Abrahamse, A., et al. 2009, Phys. Rev. D, 79, 103004 